\title{
Instrument and Operator Effects on Apple Firmness Readings
}

\author{
Laura Lehman-Salada ${ }^{1}$ \\ Department of Horticulture, The Pennsylvania State University, Fruit Research \\ and Extension Center, Box 309, Biglerville, PA 17307-0309
}

Additional index words. fruit crops, Malus domestica, maturity, postharvest handling, quality, senescence

\begin{abstract}
Three apple (Malus domestica Borkh.) cultivars of various maturity levels and flesh firmness were used to compare firmness readings obtained by three trained operators using Magness-Taylor (MT), Effegi (EF), and Electronic Pressure Tester (EPT) penetrometers. For all cultivars and operators, use of the MT resulted in the lowest mean readings, while use of the EPT operated in the MT testing mode resulted in the highest readings. Operator differences were observed for all instruments except when the electronic tester was used in the default mode. Comparisons between instruments and modes resulted in significantly different firmness readings across operators, except for the comparison of EF vs. EPT in default mode. For most comparisons, firmness variance due to instrument or mode was larger than the variance due to operator. Apple maturity did not significantly alter firmness trends due to instrument or operator. Thus, caution should be used to interpret apple firmness changes of a single lot of apples when several instruments and/or operators are used.
\end{abstract}

Flesh firmness is one of several characteristics often used to estimate fruit maturity and select proper harvest dates for apple cultivars. Because fruit softening is correlated with other physical and chemical changes during ripening, storage operators and researchers often monitor apple response to storage conditions by measuring fruit firmness over time.

Firmness determinations for apples are typically made on two peeled sites on opposite sides of the fruit equator by inserting an 11.1 $\mathrm{mm}$-diameter probe $7.9 \mathrm{~mm}$ into cortex tissue. Force is applied perpendicular to the cut surface in a smooth motion in 2 to $3 \mathrm{sec}$. In manual tests, the depth of penetration is controlled by the operator, who is trained to stop when the pared surface of the apple touches a line inscribed on the probe. Readings in poundsforce or kilograms-force are usually converted to newtons.

Although commonly referred to as a "pressure test," fruit firmness determination using a penetrometer actually measures the force required to rupture cortex parenchyma cells. Required force depends on fruit tissue resistance under the probe tip (Holt and Schoorl, 1983; LaBelle, 1981). Resistance to penetra-

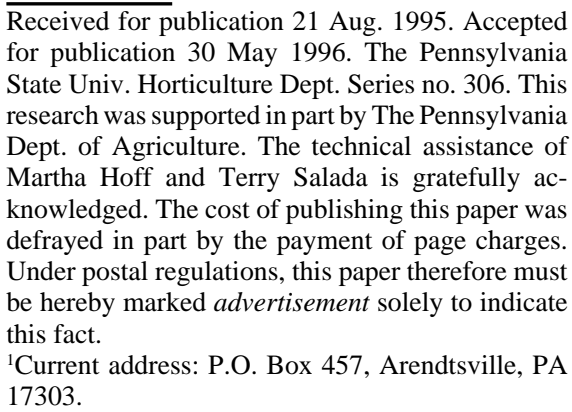

tion is determined by cell turgor, wall strength, and other factors.

Portable penetrometers, such as the Magness-Taylor tester (MT) and the Effegi firmness tester (EF) are often used in the orchard, while more sophisticated testers, such as the Electronic Pressure Tester (EPT), are frequently used in packing houses, commercial storages, and quality control laboratories. Making comparisons among firmness readings obtained by using different instruments on a single lot of fruit from a single orchard block is frequently necessary.

The MT and EF measure the maximum force required for tissue fracture (Abbott et al., 1976), while the EPT actually measures the major bioyield point from a pixel diagram of the force-deformation curve. Underquasi-static compression, the force-deformation graph becomes a jagged line with each yield event corresponding to layers of cells bursting along constant shear boundaries (Holt and Schoorl, 1983).

Many variables affect reliability of destructive firmness measurements. Lower readings are associated with apples that are large, watercored, bruised, or warm when sampled (Blanpied et al., 1978). Slow insertion speed and shallow probe penetration into the flesh may also result in artificially low readings (Blanpied et al., 1978).

The objective of this study was to compare apple flesh firmness readings obtained using three types of penetrometers in widespread use (MT, EF, and EPT testers) and to determine whether operator differences existed for each instrument. Apple-to-apple variability was minimized in instrument and operator tests by using all three methods on each apple in a set or by using two of the instruments on opposite sides of each fruit in a set.
Apples were harvested from research plantings in southern Pennsylvania according to commercial harvest dates developed for apples to be stored at least 6 months. 'Golden Delicious' were harvested in Sept. 1993 and stored in controlled-atmosphere conditions $(0 \mathrm{C}, 2 \%$ $\mathrm{O}_{2}, 0 \% \mathrm{CO}_{2}$ ) until removal for testing in Mar. 1994. 'Gala' and 'York Imperial' were harvested in Sept. and Oct. 1994, respectively, and placed in refrigerated storage at $0 \mathrm{C}$. Fruit remained in refrigerated storage no more than 2 weeks.

To provide uniform samples for testing, all apples were sized and graded before firmness determinations. Bruised, hail-injured, or stemless apples were excluded from the study. Remaining apples with similar ground color and diameters $(6.8$ to $8.1 \mathrm{~cm})$ were used to assemble 50-apple samples for instrument and operator tests. All refrigerated apples were held at $20 \mathrm{C}$ for $4 \mathrm{~h}$ before firmness testing.

Penetrometers included a hand-held MT instrument (Chatillon model 719-40; John Chatillon \& Sons, Greenesboro, N.C.), a stagemounted EF type (McCormick Fruit Pressure Tester model FT 327; McCormick Fruit Tech, Yakima, Wash.), and an EPT (model 1R; Lake City Technical Products, Kelowna, B.C.). Because the EPT can be operated in the "default mode" with speed control (SC) engaged (only determinations made at the recommended speed are accepted) or in the "MT testing mode" with SC disengaged (virtually all readings are accepted regardless of puncture speed), both modes were tested. The EPT manufacturer suggests that "MT testing mode" be used when measuring the firmness of relatively soft fruit. A previous study (unpublished) revealed no difference between apple firmness readings obtained using a hand-held EF compared to a stage-mounted EF.

Immediately before use, each penetrometer was calibrated according to the manufacturer's recommendations. Operators were instructed on how to use each device and they practiced by measuring the firmness of at least 50 apples of diverse firmness per instrument before proceeding to the study samples.

Operator test. For each cultivar, four 50apple sets were selected. One set per cultivar was randomly assigned to one of four instrument types (EF, MT, and EPT with or without $\mathrm{SC})$. Three firmness determinations were made on each fruit with one measurement made by each operator. The first puncture site on each apple was selected at random and the succeeding sites on the equator were selected such that bruised tissue from the first or second puncture site(s) was avoided. Each operator measured firmness of all 50 apples in a cultivarinstrument set before the other operators made the second and third measurements. Determinations for each set were completed in $<15$ min to minimize potentially undesirable changes in apple flesh characteristics. This protocol was followed because our previous experiments indicated that firmness variance among apples was almost always larger than 
the variance associated with repeated measurements on a single apple.

Instrument test. For this portion of the study, the firmness of eighteen 50-apple sets per cultivar was determined. Each of the three operators determined firmness separately for six sets of each cultivar. Each set was used to make one of six possible paired comparisons: EPT without SC vs. EPT with SC, EPT without SC vs. EF, EF vs. MT, EPT without SC vs. MT, EPT with SC vs. MT, and EF vs. EPT with SC. Two firmness determinations were made by the same operator on opposite sides of each apple in a set with one determination made by the first method and the second determination made by the second method. The first puncture site on each apple was selected at random and the second site was on the opposite side of the fruit equator. Measurements for each set were completed in $<9 \mathrm{~min}$.

Firmness of each apple was recorded manually for the MT and EF testers. Although the EPT recorded each reading on paper tape and automatically calculated the mean and standard deviation for each set, raw data for individual apples were analyzed.

For each operator, an analysis of variance (ANOVA) in firmness measurements was calculated for each cultivar using instrument, operator, and instrument by operator as sources of variation. An ANOVA was also performed for each cultivar within instrument comparisons to determine whether the operator influence was significant. Firmness means and standard errors were calculated for each operator and instrument within each cultivar.

For each instrument, an ANOVA was performed to determine operator and cultivar main effects and interactions for each comparison. Because linear correlation may be inappropriate for comparing two scientific methods (Altman and Bland, 1983; Peleg, 1993; Westgard and Hunt, 1973), the difference between instruments was plotted against the mean for each instrument comparison. These graphs, similar to plots of residuals, were inspected for linearity and outlier data points. After calculation of mean difference (relative bias) and standard error of each difference, Student's $t$ tests were performed using the null hypothesis that the mean difference was zero. This approach checked whether differences between instruments were independent of the value of the measurements (Altman and Bland, 1983). Linear regressions were then performed on the differences by means graphs for each instrument comparison to detect whether the mean difference in apple firmness changed as firmer fruit were sampled.

\section{Results and Discussion}

Operator tests. All sources of variation (instrument, operator, and instrument by operator interaction) in the ANOVA model for each of the three cultivars were highly significant $(P \leq 0.005)$. For each operator, the highest mean firmness readings were obtained when operators used the EPT with SC disengaged, while the lowest readings resulted from use of the MT tester (Fig. 1). The difference between EPT without SC and the MT readings for each cultivar and group of operators spanned $\approx 20$ to $25 \mathrm{~N}$, so there was evidence of a strong instrument effect relatively independent of cultivar (Fig. 1).

Operator significantly influenced firmness readings for almost all instruments and cultivar groups, except when operators used the EPT with SC engaged (Fig. 1). Our results agree with the manufacturer's claim that operator skill or technique does not influence firmness test results appreciably when the EPT is operated in the default mode (EPT SC). Bongers (1992) detected insignificant operator differences (about $3 \mathrm{~N}$ ) when measuring 'Jonagold' apple firmness with an EPT (presumably operated in the default mode), compared to an EF mounted in a drill stand or in an Instron Universal Testing machine.

Operator variability within each cultivar was not consistent from instrument to instrument (Fig. 1). One operator did not always obtain the highest or lowest mean firmness readings across all four instruments, as one might expect if some aspect of operator technique had been applied consistently when using each penetrometer.

Three possible sources of variability were suggested by the participants after this study was completed. In comparison to the other instruments, the MT was judged the most difficult to grip securely and to read clearly. Because it is much longer than the EF, finger and arm strength were challenged when using the MT, especially when very firm apples were tested. Although the operators trained and practiced with each instrument, they may have occasionally under- or overestimated the amount of force necessary for the surface of the flesh to reach the inscribed line on the MT probe.

For all instruments, the inscribed line was occasionally obscured by apple juice and operators had to wipe the front of the probe before proceeding to the next apple. An obscured line was more common with the MT tester than with the EF or EPT because the MT
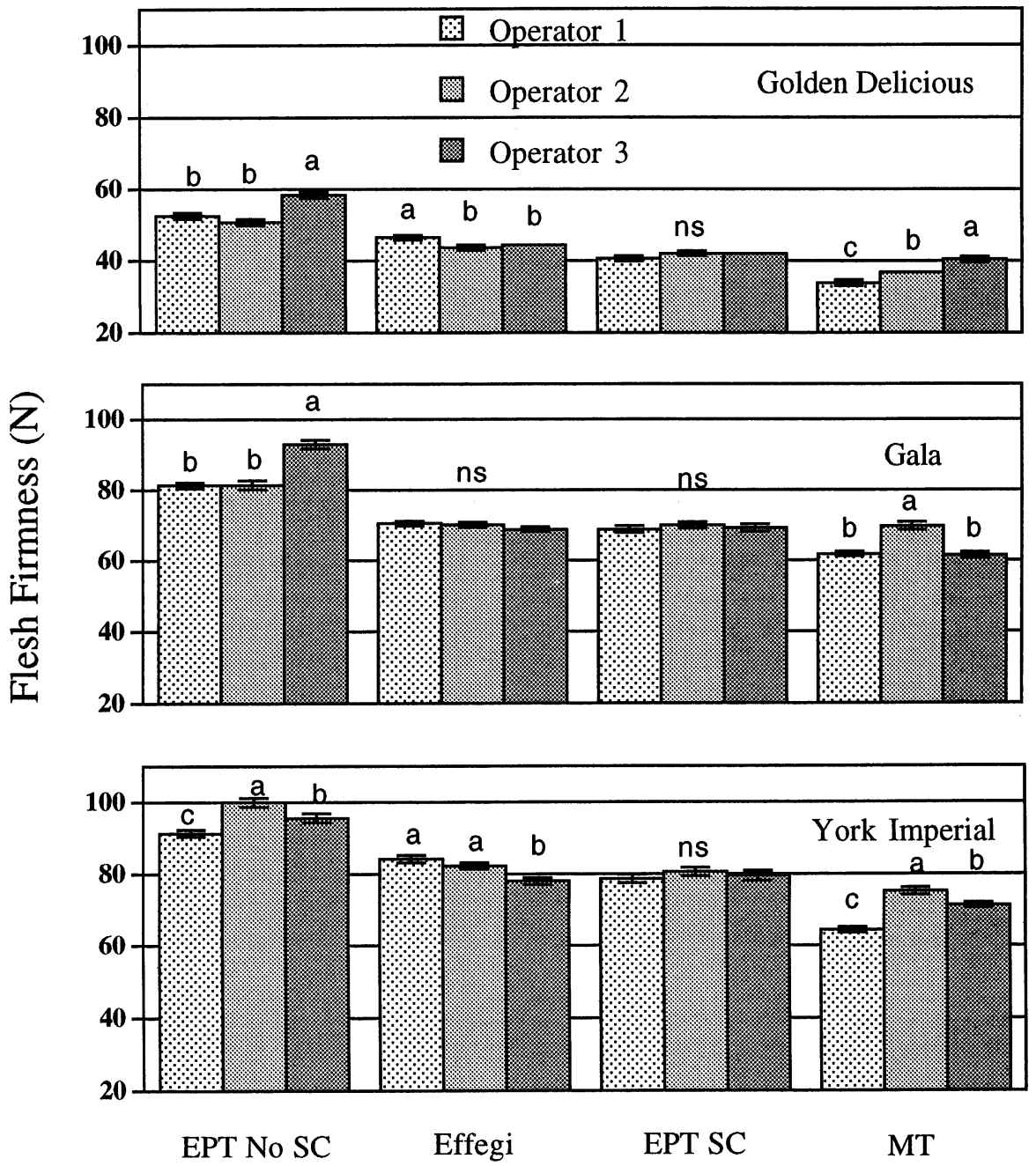

\section{Penetrometer Type}

Fig. 1. Influence of operator on flesh firmness readings obtained using an electronic tester with no speed control (EPT no SC), Effegi, electronic tester with speed control (EPT SC), or Magness-Taylor (MT) penetrometer for each of three apple cultivars. Vertical lines represent SE associated with each operator mean $(n=50)$. Mean separation of operator means for each method and cultivar by Duncan's multiple range test at $P \leq 0.05$. Nonsignificant difference between operator means denoted by NS. 
probe is not positioned to allow maximum juice drainage during use and was often farther from the operator's line of sight. Haller (1941) noted that operator technique, fruit maturity, fruit size, and other factors may influence firmness readings obtained using an MT tester. Using nonfruit samples, Nicholas (1960) found that MT readings were influenced by operator and day of use.

Stembridge et al. (1972) used an MT tester on peach [Prunus persica (L.) Batsch] fruit and reported that the SD of mean firmness changed as the crop matured. They reported that immature and overmature fruit had low SD, while fruit with intermediate firmness had the highest SD from the mean firmness. Over the firmness range in the current study, SD from the mean MT firmness across operators were similar (data not shown) with no quadratic response evident.

Another difference between these instruments involves how the data are recorded. The EF and MT scales must be read, recorded by the operator or an assistant, and then reset to zero before the next firmness measurement. The EPT does not need to be reset between determinations, and records the force required to reach the major bioyield point directly on paper tape, thus eliminating transcription error.

The amount of force used to penetrate apple flesh may be related to an inherent bias of the operator regarding expected firmness. When sampling one lot of apples with unknown firmness or with a wide range of suspected firmness, a larger sampling error is likely if operator bias is present. In this study, apples were presorted by size and suspected firmness range to intentionally reduce the SD associated with each instrument and operator (Fig. 1).

Because the depth of penetration and the rate at which force is applied are controlled by the MT or EF operator, higher variability is expected compared to firmness testing with some control over depth or rate (Abbott et al.,1976; Bourne, 1974). However, firmness of apples tested with a hand-held MT penetrometer was statistically similar to apples tested with an MT probe fixed in an Instron Universal Testing Instrument operated with a constant speed of penetration (Bourne, 1974). Abbott et al. (1976) reported a similar result when comparing apple firmness readings obtained with hand-held MT or EF testers compared to the same tester probes mounted in an
Instron ( $r=$ greater than 0.95 for all five cultivars combined).

Operator effects in instrument comparison tests. The ANOVA for firmness means for individual operators revealed that cultivar was a highly significant source of variation $(P \leq$ 0.0001 ) in each instrument comparison. Operators influenced firmness values in many comparisons, except those that included EPT with SC. This result agreed with data presented in Fig. 1, indicating an absence of operator differences for this instrument and mode. Significant cultivar by operator interactions were found for comparisons one through four listed in Table 1, while the last two comparisons were moderately influenced $(P<$ $0.01)$ or not influenced $(P<0.05)$ by cultivar and operator combined.

Correlation coefficients of the least squares regressions between instruments were relatively high (Table 1), but may not indicate a strong linear relationship between two methods. Although correlation coefficients estimate random error, they are relatively insensitive to constant and proportional error (Westgard and Hunt, 1973). Since correlation coefficients depend on the sampling error associated with each instrument and also the error resulting from the choice of experimental units (Altman and Bland, 1983), using coefficients alone to compare methods may be misleading.

Only the comparison of EPT no SC vs. MT resulted in consistently high regression line slopes for all three operators (Table 1). These two methods yielded the highest and lowest mean firmness readings, respectively (Fig. 1).

Instrument comparisons. Most of the data points for EPT no SC vs. EPT SC were above the zero force difference line, indicating that firmness readings obtained by using the former were frequently higher than those obtained with SC (Fig. 2A). The mean difference was $11 \mathrm{~N}$ with a SD of $9 \mathrm{~N}$ and a highly significant $t$ statistic. This result confirmed our earlier discovery in the operator test that the mean firmness difference between these two methods across operators was relatively large (Fig. 1).

Substantial differences between firmness readings were evident for the rest of the comparisons (Fig. 2B-F). For each of these comparisons, the $t$ statistic calculated to test the differences between instruments was $>20$, indicating a high probability of a significant difference. Mean differences were 7 to $18 \mathrm{~N}$ and there was a tendency in some comparisons for the differences to become slightly larger as firmer fruit were tested (Fig. 2A-F).

As implied in the operator test, differences were largest when comparing firmness using the EPT no SC to those obtained using the MT (Fig. 2D). Assuming a Gaussian distribution, the mean difference between methods was $>18 \mathrm{~N}$ with the $95 \%$ confidence intervals of about $20 \mathrm{~N}$. Misleading conclusions would be likely if these methods were used to measure fruit firmness of apples in the same lot at different times during the storage period.

Of the six instrument comparisons, only EF vs. EPT with SC engaged (Fig. 2F) had a mean difference near zero, with the data points almost perfectly distributed above and below the solid line representing the mean difference. Both the slope and the Y intercept of the regression line were near zero and the $t$ statistic was almost zero, indicating a nonsignificant difference between methods. Use of the $\mathrm{EF}$ and the EPT with SC engaged resulted in similar firmness values for soft, intermediate, and hard apples, although there were several outlier data points (Fig. 2F).

These results imply that firmness determinations for a given lot of apples can be made using the portable EF in the orchard and reliably compared to those made using a benchtop EPT operated in default mode. However, the firmness difference between these methods may be as large as $20 \mathrm{~N}$ (Fig. 2F), so it would be prudent to sample enough apples to account for most of the fruit-to-fruit variation. For example, Blanpied et al. (1978) suggested sampling a minimum of 60 apples per treatment when measuring firmness using an $\mathrm{EF}$ on two sites per fruit in a study with 20 fruit per replication and three replications per treatment.

Apple flesh firmness is an inherently variable characteristic. The magnitude of the firmness difference at several sites on a single apple has not been established, but preliminary studies in our laboratory indicate that firmness measured with an EPT can vary by 2 to $8 \mathrm{~N}$ when sampled at several locations around the equator. Using an MT probe mounted in an Instron, Abbott (1994) reported a relatively good simple correlation $(r=0.80)$ between firmness measured on the blush side and nonblush side of 'Delicious' apples. Future studies of instrument differences should attempt to account for instrument and apple variability.

Table 1. Least square regression equations and correlation coefficients for six comparisons of penetrometer readings made on opposite sides of each apple ( $\mathrm{n}=150$ apples per operator per comparison) as influenced by operator with data pooled across three cultivars. ${ }^{\mathrm{z}}$

\begin{tabular}{|c|c|c|c|c|c|c|c|}
\hline \multirow{2}{*}{\multicolumn{2}{|c|}{$\begin{array}{l}\text { Instrument comparison } \\
\text { for apple side }\end{array}$}} & \multicolumn{6}{|c|}{ Operator } \\
\hline & & \multicolumn{2}{|l|}{1} & \multicolumn{2}{|l|}{2} & \multicolumn{2}{|l|}{3} \\
\hline A & $\mathrm{B}$ & Equation & $\mathrm{r}^{2}$ & Equation & $r^{2}$ & Equation & $\mathrm{r}^{2}$ \\
\hline 1. EPT no SC & EPT SC & $Y=1.00 X+9.67$ & 0.85 & $Y=1.22 X-4.88$ & 0.85 & $Y=0.97 X+17.21$ & 0.82 \\
\hline 2. Effegi & EPT no SC & $Y=0.95 X+7.97$ & 0.87 & $Y=1.02 X+8.57$ & 0.85 & $Y=1.17 X+3.71$ & 0.87 \\
\hline 3. Effegi & Magness-Taylor & $Y=1.03 X+11.69$ & 0.74 & $Y=0.88 X+14.64$ & 0.85 & $Y=0.98 X+6.92$ & 0.88 \\
\hline 4. EPT no SC & Magness-Taylor & $Y=1.14 X+12.61$ & 0.84 & $Y=1.31 X-5.37$ & 0.75 & $Y=1.25 X+6.34$ & 0.75 \\
\hline 5. EPT SC & Magness-Taylor & $Y=1.06 X+8.88$ & 0.86 & $Y=0.95 X+9.82$ & 0.82 & $Y=1.03 X+4.07$ & 0.84 \\
\hline 6. Effegi & EPT SC & $Y=0.98 X+3.30$ & 0.86 & $Y=0.96 X+1.33$ & 0.88 & $Y=0.85 X+8.66$ & 0.87 \\
\hline
\end{tabular}

${ }^{2}$ EPT denotes electronic pressure tester and SC denotes speed control engaged.

${ }^{\mathrm{y}}$ First method listed in the comparison is the dependent variable. 

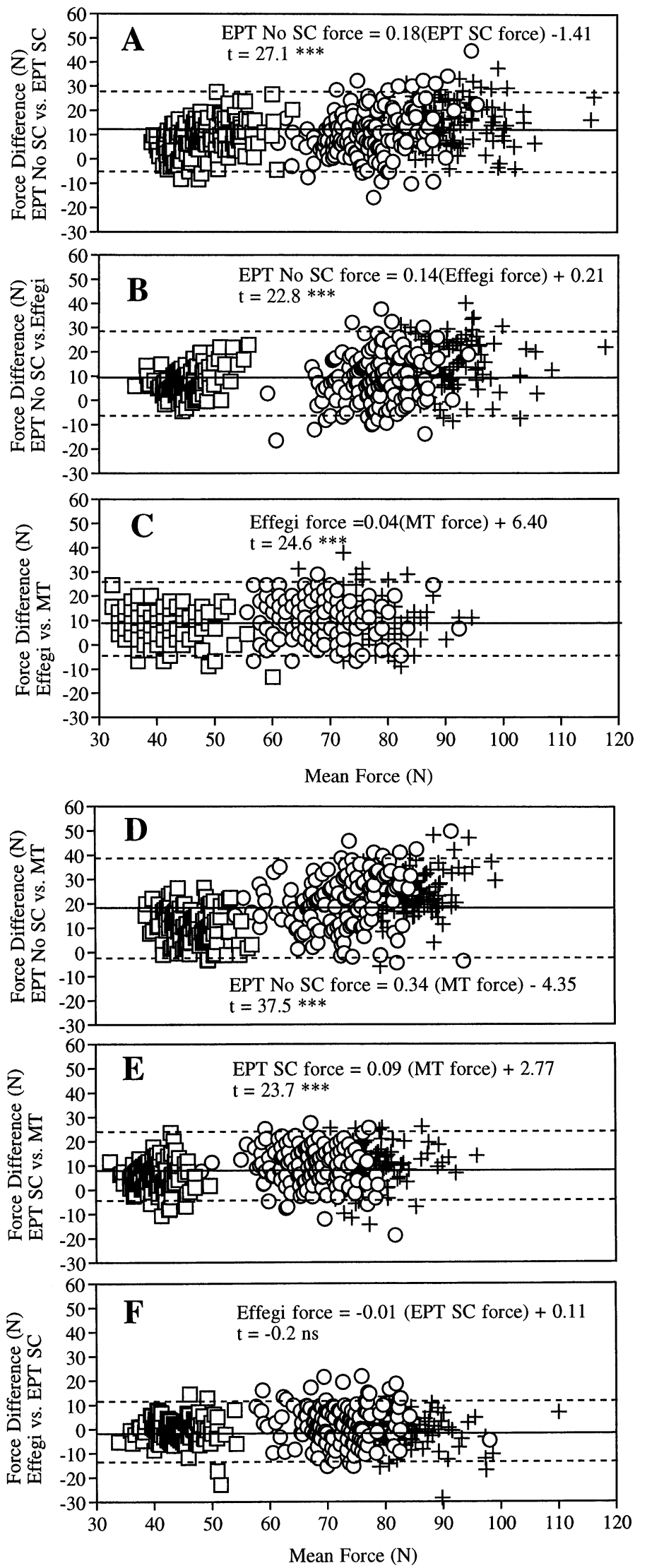

Fig. 2. Paired comparisons of flesh firmness test readings obtained using four penetrometer types and three apple cultivars of differing maturity. Penetrometer types included electronic pressure tester with speed control engaged (EPT SC) or disengaged (EPT no SC), Effegi, and Magness-Taylor (MT). (A) EPT no SC vs. EPT SC, (B) EPT no SC vs. Effigi, (C) Effigi vs. MT, (D) EPT no SC vs. MT, (E) EPT SC vs. MT, and (F) Effigi vs. EPT SC. Cultivars included 'Golden Delicious' (squares), 'Gala' (circles), and 'York Imperial' (plus signs). Solid horizontal lines denote the mean difference between two penetrometer types in each comparison, while dashed lines represent two SD from the mean reading.

\section{Conclusions}

The results of this study indicate significant differences between firmness values obtained by several penetrometers and operators. Except for one comparison, EF vs. EPT with SC engaged, differences between methods were large enough to introduce substantial errors if differing instruments and/or operators were used to measure firmness of a single lot of apples sampled over time. Our results partially reinforce the suggestion of Abbott et al. (1976), who stressed that instrument and method of measurement be clearly specified when testing apple, peach, and nectarine [Prunus persica (L.) Batsch] firmness.

If several operators must be used, conversion equations may be created to standardize firmness readings, but some accuracy will undoubtedly be lost. Although it may be timeconsuming, sampling the firmness of many apples can partially overcome problems posed by several operators. Modeling of instrument and operator combinations may be helpful for revealing minimum sample sizes necessary for apple softening studies.

\section{Literature Cited}

Abbott, J.A. 1994. Firmness measurement of freshly harvested 'Delicious' apples by sensory methods, sonic transmission, Magness-Taylor, and compression. J. Amer. Soc. Hort. Sci. 119:510 515.

Abbott, J.A., A.E. Watada, and D.R. Massie. 1976. EFFE-GI, Magness-Taylor, and Instron fruit pressure testing devices for apples, peaches, and nectarines. J. Amer. Soc. Hort. Sci. 101:698-700.

Altman, D.G. and J.M. Bland. 1983. Measurement in medicine: The analysis of method comparison studies. Statistician 32:307-317.

Blanpied, G.D., W.J. Bramlage, D.H. Dewey, R.L. LaBelle, L.M. Massey, Jr., G.E. Mattus, W.C. Stiles, and A.E. Watada. 1978. A standardized method for collecting apple pressure test data. N.Y. Food \& Life Sci. Bul. 74.

Bongers, A.J. 1992. Comparison of three penetrometers used to evaluate apple firmness. Wash. State Univ. Tree Fruit Postharvest J. 3(4):7-9.

Bourne, M.C. 1974. Comparison of results from use of the Magness-Taylor pressure tip in hand- and machine-operation. J. Texture Studies 5:105-108.

Haller, M.H. 1941. Fruit pressure testers and their practical applications. U.S. Dept. Agr. Circ. 627, Government Printing Office, Washington, D.C.

Holt, J.E. and D. Schoorl. 1983. Fracture in potatoes and apples. J. Material Sci. 18:2017-2028.

La Belle, R.L. 1981. Apple quality characteristics related to various processed products, p. 61-76. In: R. Teranishi and H. Barrera-Benitez (eds.) Quality of selected fruits and vegetables of North America. Amer. Chem. Soc. Symp. Ser. 170.

Nicholas, R.C. 1960. Some observations on the use of fruit pressure testers. Mich. Agr. Expt. Sta. Quarterly Bul. 43:312-326.

Peleg, K. 1993. Comparison of non-destructive and destructive measurement of apple firmness. J. Agr. Eng. Res. 55:227-238.

Stembridge, G.E., R.A. Baumgardner, W.E. Johnston, and L.O. Van Blaricom. 1972. Measuring uniformity of peach maturity. HortScience 74:387-389

Westgard, J.O. and M.R. Hunt. 1973. Use and interpretation of common statistical tests in methodcomparison studies. Clinical Chem. 19:49-57. 